2.3 and in the TFA group was 2.5. The mean procedure time in the TRA group was 20 and in the TFA group was 18 minutes. No patient developed neurological symptoms.

Statistical analysis were concluded using StataCorp. 2017. Stata Statistical Software: Release 15. College Station, TX: StataCorp LLC.

Conclusion Our study demonstrated no access related complications both for TRA and TFA. No significant difference was found in number of vessels catheterised and procedure time. More importantly, there were more foci of MRI DWI restriction, representing SEE, in the TRA group compared to TFA. No patient experienced associated neurological symptoms, with normal neurological examination and all were discharged immediately post recovery period. Despite of the proven benefit of the TRA for cerebral angiography, its widespread use may theoretically lead to increased frequency of embolic events during cerebral angiography, which may be of clinical significance, and this warrants further investigation.

Disclosures V. Carraro do Nascimento: None. H. Rice: 2; C; Stryker, Medtronic. L. De Villiers: 2; C; Stryker, Medtronic.

\section{LB-004 INTRAVENOUS ALTEPLASE HAS DIFFERENT EFFECTS ON THE EFFICACY OF ASPIRATION AND STENT RETRIEVER THROMBECTOMY: ANALYSIS OF THE COMPASS TRIAL}

${ }^{1} \mathrm{M}$ Mokin* ${ }^{2} \mathrm{M}$ Waqas, ${ }^{3} \mathrm{~J}$ Fifi, ${ }^{3} \mathrm{R}$ De Leacy, ${ }^{4} \mathrm{D}$ Fiorella, ${ }^{2} \mathrm{E}$ Levy, ${ }^{2} \mathrm{~K}$ Snyder, ${ }^{5} \mathrm{R}$ Hanel, ${ }^{6} \mathrm{~K}$ Woodward, ${ }^{7} \mathrm{I}$ Chaudry, ${ }^{8} \mathrm{~A}$ Rai, ${ }^{9} \mathrm{D}$ Frei, ${ }^{10} \mathrm{~J}$ Delgado Almandoz, ${ }^{11} \mathrm{M}$ Kelly, ${ }^{12} \mathrm{~A}$ Arthur, ${ }^{13} \mathrm{~B}$ Baxter, ${ }^{14} \mathrm{~J}$ English, ${ }^{15} \mathrm{I}$ Linfante, ${ }^{16} \mathrm{~K}$ Fargen, ${ }^{7} \mathrm{~A}$ Turk, ${ }^{3} \mathrm{~J}$ Mocco, ${ }^{2} \mathrm{~A}$ Siddiqui. ' University of South Florida, Tampa, FL; ${ }^{2}$ University at Buffalo, Buffalo, NY; ${ }^{3}$ Icahn School of Medicine at Mount Sinai, New York, NY; ${ }^{4}$ Stony Brook University, Ston Brook, NY; ${ }^{5}$ Baptist Medical Center, Jacksonville, FL; ${ }^{6}$ Fort Sanders Regional Medical Center, Knoxville, TN; ${ }^{7}$ Prisma Health Upstate, Greenville, SC; ${ }^{8}$ West Virginia University, Morgantown, WV; ${ }^{9}$ Swedish Medical Center, Englewood, CO; ${ }^{10}$ Abbott Northwestern Hospital, Minneapolis, MN; ${ }^{11}$ University of Saskatchewan, Saskatoon, SK, Canada; ${ }^{12}$ Semmes-Murphey Clinic, Memphis, $T N_{;}{ }^{13}$ Lehigh Valley Health Network, Allentown, PA; ${ }^{14}$ California Pacific Medical Center, San Francisco, CA; ${ }^{15}$ Baptist Cardiac and Vascular Institute, Miami, FL; ${ }^{16}$ Wake Forest Baptist Medical Center, Winston-Salem, NC

\subsection{6/neurintsurg-2021-SNIS.245}

Background There is conflicting evidence on the utility of intravenous (IV) Alteplase in patients with emergent large vessel occlusion treated with mechanical thrombectomy (MT). The goal of this study was to examine whether there were differences in procedural and technical outcomes of MT depending on whether patients received IV Alteplase prior to intervention using the COMPASS dataset.

Methods This was a post hoc analysis of the COMPASS: A trial of aspiration thrombectomy versus stent retriever thrombectomy as first-line approach for large vessel occlusion. We compared clinical, procedural and angiographic outcomes of patients with and without prior IV Alteplase administration. In order to compare outcomes in patients treated with MT according to the use of IV Altepase, only patients presenting to the emergency department (ED) of the first hospital of arrival within 4 hours of stroke symptom onset or last known normal were included in our analysis.

Results Of the 270 patients included in the COMPASS trial, $235(87 \%)$ arrived to the ED within the first 4 hours of stroke symptom onset and were eligible for the post-hoc analysis. (115 patients in the aspiration first and 120 in the SR first groups, respectively).Atrial fibrillation was more frequently seen in the MT only when compared to the combined group (65.5\% vs $38.6 \%$, respectively, $\mathrm{P}<0.001)$. Other vascular risk factors, age, NIHSS severity, and the distribution of occlusion sites showed no statistically significant difference. On univariate analysis, administration of IV Alteplase prior to MT was found to be significantly associated with favorable outcomes (modified Rankin scale [mRS] $0-2$ at 3 months; $55.6 \%$ vs $40 \%$ in the MT only group, $\mathrm{P}=0.037)$. However, on multivariate analysis only baseline (pre-stroke) mRS, admission NIHSS score and age were identified as independent predictors of favorable outcomes at 3 months. We found higher final TICI $2 b / 3$ rates in patients without the use of Alteplase prior to the aspiration first approach $(100 \%$ vs $87.9 \%$ in IV Altepase + aspiration first $\mathrm{MT}, \mathrm{P}=0.03)$. In the $\mathrm{SR}$ first group, final TICI $2 \mathrm{~b} / 3$ rates were identical in patients with and without IV Alteplase administration $(87.5 \%$ and $87.5 \%$, $\mathrm{P}=1.0)$.

Conclusions Prior administration of IV Alteplase is not associated with improved procedural or clinical outcomes in patients with anterior circulation ELVO treated with MT. Alteplase may adversely affect the efficacy of aspiration first approach, but does not seem to influence the stent retriever first approach to MT.

Disclosures M. Mokin: 1; C; NIH R21NS109575. 2; C; Cerenovus, Medtronic. 4; C; Brain Q, Endostream Serenity medical, Synchron. M. Waqas: None. J. Fifi: 2; C; Stryker, Penumbra, Microvention. 4; C; Cerebrotech, The Stroke Project. R. De Leacy: 2; C; Penumbra, Cerenovus, Siemens, Imperative Care. 4; C; Synchron, Endostream, Q’Apel. D. Fiorella: 2; C; Balt USA, Penumbra, Cerenovus, Stryker, Genentech, Shape Memory Medical, Marbelhead, Medtronic, MENTICE, Neuorogami, RAPID AI, Rapid medical, Phenox. E. Levy: 2; C; Penumbra, NextGen Biologics, Rapid Medical, Cognition Medical, Three Rivers Medical, Stryker, MedX, Endostream Medical. K. Snyder: 2; C; Penumbra, Canon Medical Systems, Medtronic, Jacobs Institute, Neurovascular Diagnostics. R. Hanel: 2; C; Penumbra, Endostream, Cerebrotech, Synchron, InNeuroCo, Medtronic, Microvention, Stryker, Cerenovus; Elum, Three Rivers. K. Woodward: None. I. Chaudry: 2; C; Medtronic, Microvention, Penumbra. 4; C; Cerenovus, Serenity Medical, Cerebrotech, Three Rivers Medical, Q'apel. A. Rai: 2; C; Penumbra, Microvention, Stryker. D. Frei: 2; C; Penumbra, Cerenovus, Stryker, Genentech, Shape Memory Medical, Siemens. J. Delgado Almandoz: 2; C; Penumbra, Medtronic. M. Kelly: 2; C; Penumbra, Medtronic, Endostream. A. Arthur: 2; C; Cerenovus, Medtronic, Microvention, Penumbra, Scientia, Siemens, Stryker. 4; C; Bendit, Cerebrotech, Endostream, Magneto, Marblehead, Neurogami, Serenity, Synchron, Triad, Vascular Simulations. B. Baxter: 2; C; Penumbra , Medtronic, Stryker, Cerenovus, Viz. ai, 880 medical, Route 92, Artio Medical. J. English: 2; C; Penumbra, Medtronic, Stryker, Route 92 Medical. I. Linfante: 2; C; Penumbra, Medtronic, Stryker, Microvention, InNeuroCo, Three Rivers. K. Fargen: None. A. Turk: 2; C; Cardinal consulting, Cerenovus, Corindus, Medtronic, Siemens, 880 medical. 4; C; Cerebrotech, Endostream. Imperative care, Three rivers medical, Vastrax, Shape memory, Synchron, Serenity medical, Blink TBI, Echovate, RIST, Apama, Q’Apel, VizAi, Early bird medical, Rapid medical, S. J. Mocco: 2; C; Imperative Care, Cerebrotech, VIseon, Endostream, Vastrax, RIST, Synchron, Viz.ai, Perflow and CVAid. 4; C; Cerebrotech, Imperative Care, Endostream, Viseon, BlinkTBI, Serenity, Cardinal Consulting, NTI, RIST, Viz.ai, Synchron. A. Siddiqui: 2; C; Amnis Therapeutics, Apellis Pharmaceuticals, Inc., Boston Scientific, Canon Medical Systems USA, Inc., Cardinal Health 200, LLC, Cerebrotech Medical Systems, Inc., 
Cerenovus, Cerevatech Medical, Inc.,. 4; C; Adona Medical, Inc., Amnis Therapeutics, Bend IT Technologies, Ltd., BlinkTBI, Inc, Buffalo Technology Partners, Inc., Cardinal Consultants, LLC, Cerebrotech Medical Systems, Inc, Cerevatech Medical.

\section{LB-005 THE INSIGHT REGISTRY: A MULTI-CENTER, MULTI-OMIC STROKE CLOT STUDY, PRELIMINARY RESULTS}

1J Fraser*, ${ }^{2}$ V Vicari, ${ }^{3} \mathrm{~A}$ Nanda, ${ }^{4} \mathrm{E}$ Cheng-Ching, ${ }^{5} \mathrm{~K}$ Woodward, ${ }^{2} \mathrm{~B}$ Rapoport, ${ }^{2} \mathrm{C}$ Kellner. ${ }^{1}$ University of Kentucky, Lexington, $\mathrm{KY} ;{ }^{2}$ Icahn School of Medicine at Mount Sinai, New York, NY; ${ }^{3}$ SSM Health, Fenton, MO; ${ }^{4}$ Premier Health - Clinical Neuroscience Institute at Miami Valley Hospital, Dayton, $\mathrm{OH}_{;}{ }^{5}$ Fort Sanders Regional Medical Center, Knoxville, TN

\subsection{6/neurintsurg-2021-SNIS.246}

Introduction Understanding clot composition and associated genomic, epigenomic, and proteomic signatures could provide insight into thrombus biology and etiology, and aid in prognosis in stroke. We report preliminary results in the first 50 subjects enrolled in this multi-omic clot collection registry. Our objective was to report our initial results from the INSIGHT Registry, a multicentered 'multi-omic' analysis of thrombi associated with acute hemorrhagic and ischemic stroke.

Abstracts LB-005 Table 1 Demographics and clinical characteristics

\begin{tabular}{|c|c|c|}
\hline & Ischemic Stroke $(\mathrm{N}=46)$ & $\begin{array}{c}\text { Hemorrhagic Stroke } \\
(\mathrm{N}=4)\end{array}$ \\
\hline Age (yxs) & $67.5(37,92) ; \mathrm{n}=46$ & $63(56,70) ; n=4$ \\
\hline \multicolumn{3}{|l|}{ Sex } \\
\hline Male & $43.5 \%(20 / 46)$ & $50 \%(2 / 4)$ \\
\hline Female & $56.5 \%(26 / 46)$ & $50 \%(2 / 4)$ \\
\hline \multicolumn{3}{|l|}{ Race } \\
\hline Asian & $0.0 \%(0 / 46)$ & $25 \%(1 / 4)$ \\
\hline Black/African American & $13 \%(6 / 46)$ & $0.0 \%(0 / 4)$ \\
\hline White & $73.9 \%(34 / 46)$ & $0.0 \%(0 / 4)$ \\
\hline Multiracial & $2.2 \%(1 / 46)$ & $0.0 \%(0 / 4)$ \\
\hline Other & $10.9 \%(5 / 46)$ & $75 \%(3 / 4)$ \\
\hline \multicolumn{3}{|l|}{ Comorbidities } \\
\hline History of Atrial Fibrillation & $10.9 \%(5 / 46)$ & $0.0 \%(0 / 4)$ \\
\hline Diabetes & $23.9 \%(11 / 46)$ & $25 \%(1 / 4)$ \\
\hline Hypertension & $73.9 \%(34 / 46)$ & $50 \%(2 / 4)$ \\
\hline Hyperlipidemia & $43.5 \%(20 / 46)$ & $50 \%(2 / 4)$ \\
\hline \multicolumn{3}{|l|}{ NIHSS on Admission } \\
\hline Minor stroke (1-4) & $15.2 \%(7 / 46)$ & $25 \%(1 / 4)$ \\
\hline Moderate stroke (5-15) & $41.3 \%(19 / 46)$ & $50 \%(2 / 4)$ \\
\hline Moderate/Severe (16-20) & $13 \%(6 / 46)$ & $0.0 \%(0 / 4)$ \\
\hline Severe stroke $(\geq 21)$ & $30.4 \%(14 / 46)$ & $0.0 \%(0 / 4)$ \\
\hline Missing & $0.0 \%(0 / 4)$ & $25 \%(1 / 4)$ \\
\hline \multicolumn{3}{|l|}{ NIHSS at Discharge } \\
\hline Recovered (0) & $17.4 \%(8 / 46)$ & $0.0 \%(0 / 4)$ \\
\hline Minor stroke (1-4) & $28.3 \%(13 / 46)$ & $0.0 \%(0 / 4)$ \\
\hline Moderate stroke (5-15) & $19.6 \%(9 / 46)$ & $75 \%(3 / 4)$ \\
\hline Moderate/Severe (16-20) & $8.7 \%(4 / 46)$ & $0.0 \%(0 / 4)$ \\
\hline Severe stroke $(\geq 21)$ & $4.3 \%(2 / 46)$ & $25 \%(1 / 4)$ \\
\hline Missing & $21.7 \%(10 / 46)$ & $0.0 \%(0 / 4)$ \\
\hline \multicolumn{3}{|l|}{ TICI Score } \\
\hline TICI $0-2 \mathrm{~A}$ & $4.3 \%(2 / 46)$ & NA \\
\hline TICI 2B & $15.2 \%(7 / 46)$ & NA \\
\hline TICI $2 \mathrm{C}$ & $10.9 \%(5 / 46)$ & NA \\
\hline TICI 3 & $69.6 \%(32 / 46)$ & NA \\
\hline LKW to Recanalization (Minutes) & $310(119,7230) ; \mathrm{n}=42$ & NA \\
\hline
\end{tabular}

Material and Methods Subjects age $\geq 18$ years, treated frontline with the Penumbra System for thrombectomy or the Artemis Neuro Evacuation device for minimally invasive endoscopic intracerebral hematoma evacuation as standard of care are included. Patient demographics, medical history, radiographic, and procedural information are collected in conjunction with extracted clot and concurrent extracranial arterial blood. Upon collection, clots were placed in RNAlater for preservation of protein and RNA and peripheral blood in K2EDTA tubes, allowing sample shipment at ambient temperature. RNA isolated from clot and blood were quality controlled by TapeStation and quantified by QuBit. Protein is quantified by bicinchoninic acid (BCA) assay. Genomic DNA isolated from peripheral blood is sequenced using the Illumina Infinium Global Screening Array. Follow-up clinical assessments occur at discharge and/or day 7, and at 90 days post-procedure.

Results 50 subjects were enrolled from 8 active sites between 02/2021 and 06/2021 (table 1). As expected, most enrollments were in ischemic stroke thrombectomy (46) versus intracranial hemorrhage (4), reflecting the relative incidence and utilization of those procedures. The initial dataset provided reflects realworld results consistent with published thrombectomy studies. RNA isolated from clots had an average RNA integrity score (RIN) of $4( \pm 1)$ ranging from 2-8, while RNA isolated from sytemic arterial blood averaged $5( \pm 2)$ with a range from 2-9 with 1 sample fail $(n=48)$. Total protein isolated from clots averaged 3028ug $( \pm 5407)$ ranging from 32-23,451ug $(n=24)$, while total protein isolated from blood averaged 2026ug $( \pm 1265)$ ranging from $241-5334(n=48)$.

Conclusion These preliminary results demonstrate the realworld applicability of the INSIGHT study to typical stroke thrombectomy population. Furthermore, the tissue processing protocols are providing high-quality RNA and protein for analysis, which is currently underway.

Disclosures J. Fraser: 1; C; Penumbra, University of Kentucky. 2; C; Stream Biomedical, Medtronic. 4; C; Cerelux, Fawkes Biotechnology. J. Vicari: None. A. Nanda: None. E. ChengChing: None. K. Woodward: None. B. Rapoport: None. C. Kellner: None.

\section{LB-006 COLLATERAL IMAGING OF STROKE PATIENTS IN THE LATE WINDOW PRIOR TO ENDOVASCULAR THROMBECTOMY RESULTS FROM A POOLED INDIVIDUAL PATIENT-LEVEL MULTICENTER ANALYSIS}

${ }^{1} \mathrm{M}$ Almekhlafi* ${ }^{2}{ }^{2} \mathrm{~J}$ Thornton, ${ }^{3} \mathrm{I}$ Casetta, ${ }^{1} \mathrm{M}$ Goyal, ${ }^{4} \mathrm{~N}$ Stefania, ${ }^{5} \mathrm{D}$ Herlihy, ${ }^{6} \mathrm{E}$ Fainardi, ${ }^{5} \mathrm{~S}$ Power, ${ }^{7} \mathrm{~V}$ Saia, ${ }^{5} \mathrm{~A}$ Hegarty, ${ }^{8} \mathrm{G}$ Pracucci, ${ }^{1} \mathrm{~A}$ Demchuk, ${ }^{9} \mathrm{~S}$ Mangiafico, ${ }^{5} \mathrm{~K}$ Boyle, ${ }^{4} \mathrm{P}$ Michel, ${ }^{1} \mathrm{~F}$ Bala, ${ }^{1} \mathrm{R}$ Gill, ${ }^{10} \mathrm{~A}$ Kuczynski, ${ }^{1} \mathrm{~A}$ Ademola, ${ }^{1} \mathrm{M}$ Hill, ${ }^{11} \mathrm{D}$ Toni, ${ }^{12} \mathrm{~S}$ Murphy, ${ }^{13} \mathrm{~B}$ Kim, ${ }^{1} \mathrm{~B}$ Menon. ${ }^{1} \mathrm{U}$ of Calgary, Calgary, AB, Canada; ${ }^{2}$ Neuroradiology, Beaumont Hospital, Dublin, Ireland; ${ }^{3}$ Clinica Neurologica, University of Ferrara, Ferrara, Italy; ${ }^{4}$ Lausanne University Hospital, Lausanne, Switzerland; ${ }^{5}$ Beaumont Hospital, Dublin, Ireland; ${ }^{6}$ University of Florence, Florence, Italy; ${ }^{7}$ Santa Corona Hospital, Pietra Ligure (SV), Italy; ${ }^{8}$ Careggi University Hospital, Florence, Italy; ${ }^{9}$ IRCCS Neuromed, Pozzilli (IS), Italy; ${ }^{10} \mathrm{U}$ of Toronto, Toronto, ON, Canada; ${ }^{11}$ Sapienza University Hospital, Rome, Italy; ${ }^{12}$ The Mater Misericordiae University Hospital, Dublin, Ireland; ${ }^{13}$ Seoul National University Bundang Hospital, Seoul, Korea, Republic of

\subsection{6/neurintsurg-2021-SNIS.247}

Background Collateral assessment using CT angiography (CTA) is promising to increase the eligibility for endovascular thrombectomy (EVT) in the late window (6-24 hours). The outcome of these patients compared to those selected using perfusion imaging is not clear. We aimed to describe the 